\title{
EFEITO DA KINESIO TAPING NO DESEMPENHO DO SALTO HORIZONTAL E VERTICAL EM ATLETAS RECREACIONAIS DE VOLEIBOL
}

Lucas Meireles Matos

Giulia Calandrini Pestana de Azevedo Janine Brasil de Araújo Moraes

Graduação em Fisioterapia pela Universidade do Estado do Pará (UEPA), Brasil.

\section{Ana Julia Cunha Brito}

Fisioterapeuta. Mestre em Desenvolvimento e Meio Ambiente Urbano pela Universidade da Amazônia, Docente da Faculdade Cosmopolita, Belém (PA), Brasil.

\section{Biatriz Araújo Cardoso}

Fisioterapeuta, Doutora em Ciências pelo Programa de Medicina Tropical pelo Instituto Oswaldo Cruz/ FIOCRUZ, Docente do Departamento de Ciências do Movimento Humano da Universidade do Estado do Pará, Belém (PA), Brasil.

\section{George Alberto da Silva Dias}

Fisioterapeuta, Doutor em Doenças Tropicais pelo NMT/UFPA/PA, Docente do Departamento de Ciências do Movimento Humano da Universidade do Estado do Pará, Belém (PA), Brasil.
RESUMO: O objetivo deste trabalho concentra-se em avaliar os efeitos da Kinesio Taping na potência dos membros inferiores após 24, 48 e 72 horas de aplicação da bandagem, bem como 24 horas após a sua retirada. Trata-se de estudo experimental, com 40 atletas, divididos em grupo placebo (GP) e grupo Kinesio Taping (GKT). Foram avaliados o Single-Leg Hop Test, salto em distância e o teste de impulsão vertical, durante cinco dias consecutivos. Utilizou-se os testes ANOVA, $\mathrm{t}$ Student e Mann-Whitney $(p \leq 0,05)$. No grupo GKT não houve aumento da potência dos membros inferiores ao longo dos dias de uso do KT. Porém, quando comparado ao GP, mostrou-se superior para os três saltos. No Single-Leg Hop Test houve melhora após 72 horas de aplicação e 24 horas após a retirada do KT. Para o salto em distância houve melhora durante 24, 48 e 72 horas após a aplicação do KT e 24 horas após a sua retirada. E o teste de impulsão vertical apenas melhorou após 24, 48 e 72 horas de aplicação do KT. Assim, o KT não mostrou melhora da potência durante os dias, porém apresentou superioridade quando comparado ao GP.

PALAVRAS-CHAVE: Fita Atlética; Força Muscular; Voleibol.

\section{THE EFFECT OF KINESIO TAPING IN THE PERFOR- MANCE OF HORIZONTAL AND VERTICAL JUMPS IN VOLLEYBALL ATHLETES}

\begin{abstract}
The effects of Kinesio Taping in the strength of the lower members are evaluated after 24, 48 and 72 hours of bandage application and after 24 hours after release. Current experimental study involved 40 athletes, divided into a placebo group (PG) and Kinesio Taping Group (KTG). The Single-Leg Hop Test, distance jump and the vertical impulse test were assessed during five consecutive days. ANOVA, Student's $s$ test and Mann-Whitney test were employed ( $\mathrm{p} \leq 0.05)$. There was no increase in the strength of the lower members in the KTG during the days in which KT was employed. When compared to PG, it was better for the three jumps. In the case of Single-Leg Hop Test, an improvement occurred during 24, 48 and $72 \mathrm{~h}$ of KT application and $24 \mathrm{~h}$ after release. In the case of the vertical impulse, improvement occurred only after 24, 48 and 72 hours of KT application. KT failed to improve strength even though higher rates occurred when compared to $P G$ results.
\end{abstract}

KEY WORDS: Athletic Tape; Muscle Strength; Volleyball.
Autor correspondente:

George Alberto da Silva Dias

georgealbertodias@yahoo.com.br 


\section{INTRODUÇão}

O voleibol é atualmente apontado como o segundo esporte em popularidade no Brasil, em virtude dos consecutivos títulos que suas seleções, tanto as de base quanto as adultas dos dois gêneros, vêm alcançando ao longo das últimas décadas ${ }^{1}$.

Nesta modalidade existe uma série de valências físicas e gestos motores que são utilizados pelos atletas, fazendo com que estes se destaquem no âmbito esportivo e delimitando diferentes funções dentro da equipe, de acordo com as especialidades de cada jogador ${ }^{1-3}$. Estatisticamente, o maior número de pontos em um jogo de voleibol ocorre de duas formas, por meio do salto oblíquo e vertical rente a rede ${ }^{2}$.

Ferreira et al. ${ }^{4}$ postulam que um dos principais, se não o principal, gesto motor utilizado na modalidade é a impulsão vertical do atleta e que unindo à altura do mesmo pode ser o principal diferencial durante o jogo. O voleibol é um esporte que apresenta um alto grau de potência muscular, principalmente sobre os membros inferiores. Visto que os movimentos exigidos durante os saltos envolvem as articulações do tornozelo, joelho, quadril e ombros. O alinhamento ótimo deste jogo articular se faz necessário, tanto na fase preparatória quanto na fase propulsiva, pois a aceleração gerada estimula a produção de força e proporciona a realização de um bom salto5.

Com isso, o bloqueio ou mesmo o ataque à frente da linha de três metros pode ser caracterizado por tal gesto esportivo. Entre outras ações no voleibol, se observa movimentos combinados entre corridas curtas e saltos, como as passadas e ataques a partir desta linha, caracterizando as impulsões horizontais e movimentos oblíquos ${ }^{5}$.

Todos estes saltos realizados durante o voleibol necessitam de rapidez e força, e se enquadram na denominação de um esforço explosivo, potente, sobre a ação do chamado ciclo de alongamento - encurtamento (CAE), o qual proporciona um aumento do rendimento motor em movimentos que utilizam ações musculares excêntricas, seguidas imediatamente das concêntricas ${ }^{3,6}$.

Um dos grupos musculares que realiza este ciclo é o tríceps sural, músculo de impulsão e responsável pela flexão plantar do tornozelo, compondo em torno de $22 \%$ dos movimentos de saltos do voleibol. Dentre este conjunto muscular, os gastrocnêmios são os mais superficiais do compartimento posterior da perna e, consequentemente, facilmente manipuláveis. Com a predominância de fibras do tipo II, denominadas de contração rápida, proporcionam a produção de movimentos rápidos durante a corrida e o salto ${ }^{7}$.

$\mathrm{Na}$ tentativa de se buscar aumento da potência muscular, através da somação de estímulos para gerar contrações cada vez mais rápidas e eficazes, na área da reabilitação esportiva existem recursos capazes de melhorar o desempenho muscular e/ou, até mesmo, acelerar o processo de reabilitação dos atletas. Dentre eles, as bandagens elásticas são uma alternativa encontrada para promover e favorecer a manutenção de estímulos musculares durante a prática de atividades esportivas ${ }^{2,3}$.

Em meio às bandagens utilizadas no esporte, encontra-se a Kinesio Taping (KT), que foi desenvolvido em 1973 por Kenso Kase no Japão, e obteve importante relevância em 2008, durante os Jogos Olímpicos de Pequim, na China, sendo aplicado em diversos atletas de alto rendimento, o que garantiu investimentos no tratamento e visibilidade às pesquisas voltadas para o esporte ${ }^{6}$.

A bandagem é livre de látex, com capacidade adesiva acrílica ficando ativada pelo calor do corpo, feita de fio elástico de polímero envolto por fibras de algodão $0^{4,6,7}$. Suas características superam as outras bandagens comumente utilizadas, pois promove secagem mais rápida, maior tempo de uso, ser fina e mais elástica (com alongamento/estiramento longitudinal de $55 \%$ a $60 \%$ da sua posição de repouso ou elasticidade total de 120 a 140\% $)^{8}$.

Este método tornou-se uma alternativa viável na prática esportiva, pois suas propriedades, com destaque à facilitação dos movimentos, pode aprimorar os estímulos neuromusculares capazes de auxiliar a velocidade de resposta e o desempenho da contração muscular. Alguns autores demonstram que o uso de bandagens dá subsídios para a correção da função muscular. Com isso, desencadeiam ações sobre os proprioceptores cutâneos, possibilitando ações sobre os movimentos e o posicionamento articular. Atua no alinhamento das fibras musculares, a partir da amenização de espasmos, redução da dor, por meio de vias neurais e no fortalecimento de músculos fracos $^{9,10}$ 
No entanto, existem autores que discordam da ação efetiva do KT sobre a melhora na performance muscular $^{11}$, relatam que este método pode não ser capaz de modular um incremento da força muscular em atletas saudáveis, mas pode produzir efeito positivo na redução da fadiga muscular. Outro estudo ${ }^{12}$, de revisão sistemática, encontrou resultados interessantes sobre os efeitos do KT. A grande maioria dos estudos não revelou que os efeitos do KT fossem superiores ao grupo controle. E quando observou esta superioridade, os efeitos foram pequenos e provavelmente com pouca significância clínica ou ensaios clínicos com baixa qualidade.

Pesquisas referentes à saúde do atleta são importantes, principalmente as que almejam melhorar a performance física, tanto através de análises subjetivas (qualitativas) quanto análises mais objetivas (quantitativas). Entender o gesto esportivo de uma determinada modalidade permite desenvolver estratégias que possam aprimorar a técnica do esporte e o desempenho morfofuncional do praticante e assim gerar grandes resultados no processo de prevenção de lesões ${ }^{13}$.

Investigar variáveis cinéticas e cinemáticas, por meio do KT, no desempenho esportivo, permite elucidar as vantagens de utilização e aplicação da técnica em atletas saudáveis. Sendo assim, o objetivo deste estudo foi avaliar os efeitos da Kinesio Taping na potência dos membros inferiores após 24, 48 e 72 horas de aplicação da bandagem, bem como 24 horas após a sua retirada, a fim de buscar novos entendimentos acerca da bandagem e suas repercussões no desempenho funcional de atletas recreacionais de voleibol.

\section{METODOLOGIA}

\section{ASPECTOS ÉTICOS}

A pesquisa teve início após aprovação do Comitê de Ética em Pesquisa da Universidade do Estado do Pará (UEPA) (Parecer $\mathrm{n}^{\circ}$ 2.069.831 de 2017). Trata-se de uma pesquisa experimental, seguindo as normas da Resolução 466/12 do Conselho Nacional de Saúde, relativa à pesquisa com seres humanos. Todos os participantes assinaram o Termo de Consentimento Livre e Esclarecido.

\section{AMOSTRA}

Participaram do estudo 40 atletas universitários (universo) praticantes recreacionais de voleibol, da UEPA, e avaliados no período de setembro de 2016 a maio de 2017. Sendo divididos em dois grupos, o primeiro denominado grupo Kinesio Taping (GKT) e o segundo como grupo placebo (GP), ambos com 20 participantes, selecionados por meio da amostragem probabilística aleatória simples, na qual à medida que os atletas foram sendo incluídos no estudo, eles se destinavam a um grupo específico.

Como critérios de inclusão optou-se por indivíduos na faixa etária de 18 a 35 anos, gênero masculino, sem história de dano tecidual nos músculos gastrocnêmios ou que estivessem em tratamento médico ou fisioterapêutico por lesões nos membros inferiores ou superiores nos últimos seis meses antes do período de avaliação da pesquisa, com o mesmo nível de treinamento e com a mesma intensidade de treinos praticados na universidade.

Foram excluídos atletas que apresentassem sintomas de dores musculares tanto em membros inferiores quanto superiores, processo alérgico à bandagem, indivíduos que tivessem ingerido suplementos musculares durante a fase experimental do trabalho, bem como apresentassem diferença no comprimento dos membros inferiores maiores que $1 \mathrm{~cm}$ do lado dominante em relação ao contralateral, portadores de deficiência física ou de sequela de doença neurológica e voluntários que não comparecessem aos testes propostos pela pesquisa.

\section{PROCEDIMENTOS DE APLICAÇÃO DO KT}

Utilizou-se na pesquisa a Kinesio Tex Gold Tape ${ }^{\circledR}$ (Kinesio Holding Corporation, Albuquerque, NM), uma bandagem a prova d'água, porosa e adesiva. Os participantes receberam orientações quanto a realizar suas atividades diárias sem restrições, como por exemplo, molhar na hora de tomar banho.

Os dois grupos utilizaram a bandagem em formato "Y". Esta teve sua aplicação sobre a região do músculo gastrocnêmio em ambos os membros inferiores, estando os sujeitos em posição ortostática realizando dorsiflexão ativa do tornozelo ${ }^{14}$. No grupo GKT buscou-se a facilitação muscular utilizando a técnica Paper Off ${ }^{14,15}$. Somente na região do tendão calcâneo adotou-se tensão média de 
50\%. O GP recebeu a bandagem também no mesmo sentido do grupo GKT, porém sem nenhuma tensão. Em ambos os grupos nenhum dos atletas tinha conhecimento de qual técnica estava sendo aplicada.

Foram aplicadas bandagens âncoras, uma na linha poplítea do joelho e outra no médio pé, dispostas na horizontal para fixar e garantir a permanência do material na realização dos testes. Para garantir a credibilidade do procedimento adotado no estudo, um pré-teste foi realizado a fim de estabelecer a eficiência do examinador na aplicação da técnica de bandagem e, assim, detectar previamente qualquer intercorrência. Durante todo o processo de coleta dos dados a bandagem foi aplicada por um único pesquisador habilitado.

\section{PROCEDIMENTO DE AVALIAÇÃO}

A escolha dos testes funcionais utilizados baseou-se em estudos sobre os tipos de salto mais difundidos na literatura atualizada, com ampla utilização, aceitação e importância, e que apresentavam altos graus de confiabilidade para reprodução, conforme verificado no estudo de Branco ${ }^{16}$.

Três testes específicos, dois saltos horizontais e um vertical, fizeram parte da avaliação, e foram usados com o objetivo de verificar o rendimento físico dos atletas durante os dias e comparar os grupos sob a influência da bandagem elástica. $\mathrm{O}$ resultado utilizado incidia sobre as maiores distâncias atingidas, considerando para análise apenas o maior valor obtido após três tentativas consecutivas com repouso passivo entre elas ${ }^{17}$.

Antes do início da coleta de dados, todos os sujeitos pesquisados foram familiarizados com os procedimentos referentes aos saltos, a fim de garantir uma execução adequada e segura. Vale ressaltar que a aplicação e validação desses testes foram realizadas pelo mesmo pesquisador, com o objetivo de evitar viés da pesquisa. Uma fita métrica serviu como instrumento de aferição da metragem atingida.

O primeiro salto horizontal foi o Single-Leg Hop Tes ${ }^{18}$ no qual os atletas foram instruídos a saltar o mais longe possível em apoio unipodálico com o membro dominante (Figura 1). A distância do salto foi medida em centímetros, a partir da posição inicial do teste até o ponto de apoio. Além disso, os membros superiores estavam voltados para trás, com rotação interna de ombro e flexão do cotovelo.

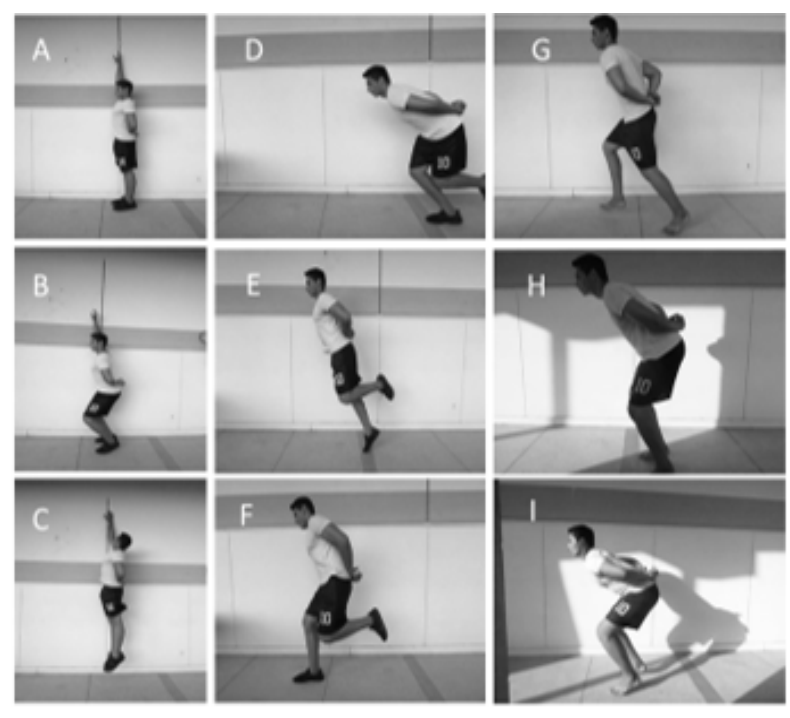

Figura 1. Imagens A, B e C - Teste de impulsão vertical; Imagens D, E e F - Single-Leg Hop Test; Imagens G, H e I - Salto em distância

Os membros superiores permaneceram em cadeia cinética fechada quando as mãos estavam seguras umas às outras durante o salto priorizando as ações concêntricas dos músculos dos membros inferiores, sobretudo o gastrocnêmio, responsável pela impulsão e estabilidade do atleta na ação realizada ${ }^{19}$, esta modificação dos membros superiores também esteve presente no salto em distância.

O segundo teste horizontal aplicado, teste de salto em distância ${ }^{3}$ (Figura 1), também foi aferido em centímetros, a partir da demarcação inicial do salto. Porém, houve instrução aos atletas para percorrerem três metros até a linha do salto demarcado. Durante o percurso os participantes receberam orientações para realizar o salto conforme a prática esportiva usada no voleibol, com desvios laterais e passadas largas a fim de ganhar impulso.

Por fim, realizou-se o teste de impulsão vertical ${ }^{20}$ (Figura 1), para este teste se fez necessário fixar uma fita métrica na parede, verticalmente. $\mathrm{O}$ atleta ficava disposto lateralmente a ela, com os pés apoiados ao solo e com o braço dominante estendido acima da cabeça, a fim de demarcar o ponto mais alto alcançado pelo terceiro dedo sinalizando o começo da mensuração dos valores. O salto iniciou a partir da flexão dos membros inferiores e executou-se a impulsão contra o movimento até tocar o ponto mais alto na parede. Para tal marcação, foi utilizado pó de giz na extremidade do terceiro dedo. O valor foi calculado pela diferença de alturas, da maior altura alcançada (com salto) e a da altura parado, em centímetros.

A coleta dos dados ocorreu durante cinco dias 
consecutivos, buscando avaliar os efeitos após 24, 48 e 72 horas de aplicação da bandagem, bem como 24 horas após a retirada da bandagem e se deu da seguinte forma: no primeiro encontro foram realizados os testes, e, logo após, aplicou-se a bandagem. Nos dois dias seguintes, os atletas submeteram-se novamente à bateria de saltos propostos no estudo. Já no quarto dia, 72 horas após a aplicação da bandagem, os pesquisados refizeram os testes e retiraram a bandagem. No quinto dia de avaliação, houve a mensuração dos últimos valores referentes à bateria de exames, ou seja, 24 horas após a retirada da bandagem. Os sujeitos realizavam os testes sempre no mesmo turno dos dias de avaliação. A Figura 2 mostra os procedimentos metodológicos realizados na pesquisa.

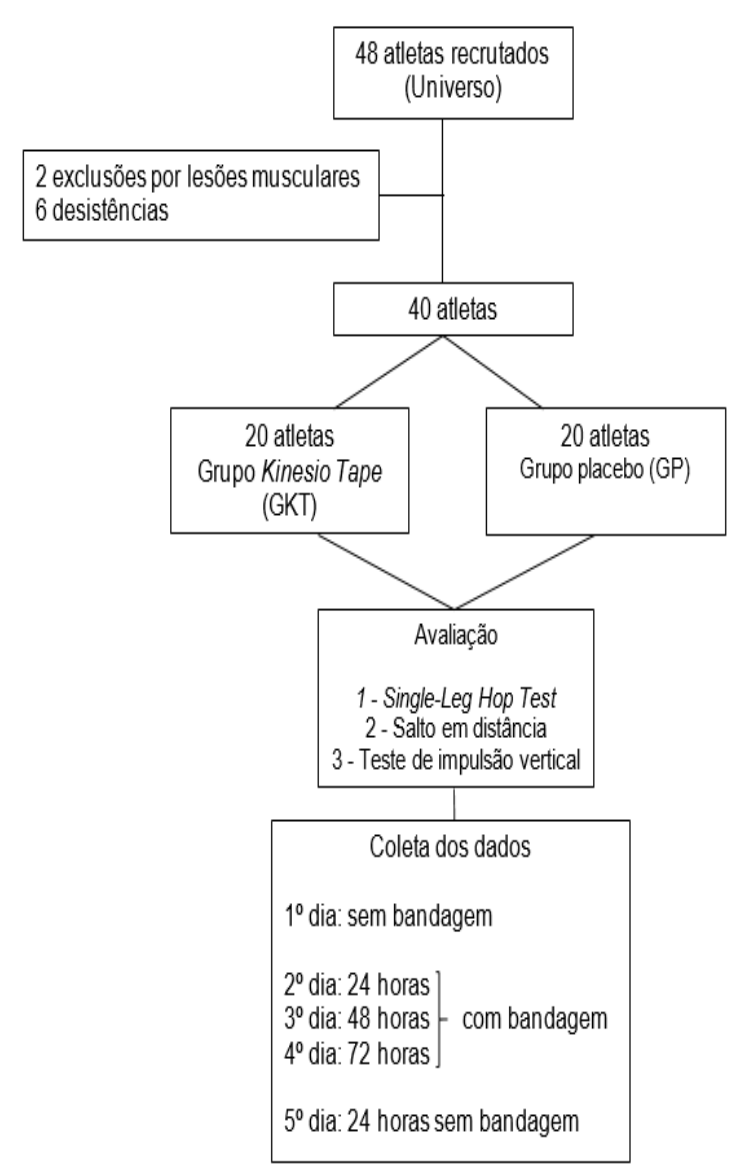

Figura 2. Fluxograma dos procedimentos metodológicos da pesquisa

\section{ANÁLISE ESTATÍSTICA}

As variáveis numéricas foram apresentadas por meio de medidas de tendência central (média) e dispersão (desvio padrão). Verificou-se a normalidade dos dados empregando o teste D'Agostino, onde estes reve- laram-se com distribuição normal e outros não normal. Assim, na análise estatística inferencial utilizou-se o teste ANOVA (paramétrico) para avaliar a variância dos dados, o teste Mann-Whitney (não paramétrico) e o teste $t$ Student (paramétrico) comparando variáveis numéricas entre dois grupos. Os tamanhos dos efeitos foram classificados usando o d de Cohen com seguintes critérios: trivial $(<0,35)$, pequeno $(0,35-0,80)$, moderado $(0,80-1,50)$ e grande $(>1,50)^{21}$. Adotou-se o nível de significância de $\mathrm{p}$ $\leq 0,05$, utilizando o software BioEstat 5.0.

\section{RESULTADOS}

Primeiramente, participaram da avaliação inicial 48 indivíduos, no entanto houve duas exclusões de atletas devido a lesões musculares (distensões) e quatro desistências. Assim, concluíram a pesquisa 40 atletas, 20 do grupo GKT e 20 do grupo GP. A média de idade do grupo GKT foi de 20,5 anos, altura média de 174,8 centímetros e massa corporal média de 74,7 kg. Já no grupo GC a média de idade esteve em 20,8 anos, 177,4 centímetros e 76,8 $\mathrm{kg}$. Não houve diferença nas variáveis idade $(\mathrm{p}=0,63)$, altura $(\mathrm{p}=0,14)$ e massa corporal $(\mathrm{p}=0,58)$ entre os grupos estudados, como mostra a Tabela 1. Este resultado demonstra que as amostras são semelhantes para estas variáveis. $\mathrm{E}$, diante deste fato, não representam um viés de equívoco nas análises dos saltos dos atletas. Portanto, todos os participantes apresentavam semelhanças na performance física e intensidade de treinamento por fazerem parte da seleção de vôlei da universidade.

Tabela 1. Distribuição demográfica dos atletas do grupo kinesio taping (GKT) e grupo placebo (GP) avaliados no período de setembro de 2016 a maio de 2017, Belém - Pará

\begin{tabular}{|c|c|c|c|c|}
\hline Variáveis & GKT & GP & $\begin{array}{c}\text { d de } \\
\text { Cohen }\end{array}$ & $\begin{array}{c}\text { *p-va- } \\
\text { lor }\end{array}$ \\
\hline Idade & $\begin{array}{c}20,5 \pm \\
1,98\end{array}$ & $\begin{array}{c}20,8 \pm \\
2,50\end{array}$ & 0,1 & 0,63 \\
\hline Estatura $(\mathrm{cm})$ & $\begin{array}{c}174,8 \pm \\
5,99\end{array}$ & $\begin{array}{c}177,4 \pm \\
5,21\end{array}$ & 0,4 & 0,14 \\
\hline $\begin{array}{c}\text { Massa corporal } \\
(\mathrm{kg})\end{array}$ & $\begin{array}{c}74,7 \pm \\
8,03\end{array}$ & $\begin{array}{c}76,8 \pm \\
14,74\end{array}$ & 0,1 & 0,58 \\
\hline
\end{tabular}

No grupo GKT observa-se que não houve diferença estatística no rendimento físico dos atletas nos 
três saltos avaliados. Ou seja, com o passar dos dias o uso da bandagem não levou ao aumento significativo dos valores obtidos nos saltos horizontais e vertical. No grupo GP, apesar de não ter sido utilizada técnica de facilitação muscular, também não foi observada essa variação nos testes utilizados, conforme mostra a Tabela 2.

Tabela 2. Média do desempenho (em $\mathrm{cm})$ dos grupos nos testes avaliados ao longo de cinco dias

\begin{tabular}{|c|c|c|c|c|c|c|}
\hline \multirow{2}{*}{ Dias } & \multicolumn{3}{|c|}{ GKT } & \multicolumn{3}{|c|}{ GP } \\
\hline & S1 & S2 & S3 & S1 & S2 & S3 \\
\hline $1^{\circ} \mathrm{dia}$ & 169,9 & 223,1 & 101,7 & 163,8 & 210,6 & 96,59 \\
\hline $2^{\circ}$ dia & 179,1 & 235,6 & 102,5 & 168,1 & 204,4 & 97,05 \\
\hline $3^{\circ}$ dia & 182,2 & 233,4 & 103,5 & 173,6 & 204,6 & 97,58 \\
\hline $4^{\circ}$ dia & 187,7 & 233,9 & 103,7 & 170,3 & 210,3 & 98,19 \\
\hline $5^{\circ}$ dia & 185,1 & 228,7 & 101,7 & 171,4 & 206,5 & 98,23 \\
\hline $\begin{array}{l}\text { *p- } \\
\text { valor }\end{array}$ & 0,2097 & 0,8491 & 0,9447 & 0,6972 & 0,8860 & 0,9281 \\
\hline
\end{tabular}

GKT (Grupo Kinesio Taping); GP (Grupo placebo).

S1(Single-Leg Hop Test); S2 (salto em distância); S3 (teste de impulsão vertical).

Nota: Teste ANOVA, ${ }^{*} \mathrm{p} \leq 0,05$.

Quando comparado o grupo GKT com o GP buscando diferença no desempenho funcional dos atletas, percebeu-se superioridade do grupo GKT em algumas situações. A técnica de facilitação muscular obteve resultados significativos nos valores dos saltos dos atletas em comparação com aqueles que utilizavam a bandagem, porém sem técnica específica.

Com relação ao Single-Leg Hop Test, observou-se que somente no $4^{\circ}$ e $5^{\circ}$ dia houve diferença significativa em relação ao desempenho dos atletas nos valores obtidos do salto, onde o grupo GKT obteve resultados superiores quando comparados aos do grupo GP, conforme mostra a Tabela 3.

Tabela 3. Comparação das médias de desempenho (em cm) no salto Single-Leg Hop Test entre o grupo kinesio taping (GKT) e o grupo placebo (GP)

\begin{tabular}{|c|c|c|c|c|}
\hline \multirow{2}{*}{ Dias } & \multicolumn{2}{|c|}{ Single-Leg Hop Test } & \multirow{2}{*}{$\begin{array}{c}\text { d de } \\
\text { Cohen }\end{array}$} & \multirow{2}{*}{ *p-valor } \\
\hline & GKT & GP & & \\
\hline $1^{\circ}$ dia & $169,9 \pm 38,36$ & $163,8 \pm 25,67$ & 0,1 & 0,51 \\
\hline $2^{\circ} \mathrm{dia}$ & $179,1 \pm 34,87$ & $168,1 \pm 21,42$ & 0,3 & 0,15 \\
\hline $3^{\circ}$ dia & $182,2 \pm 33,24$ & $173,6 \pm 20,71$ & 0,3 & 0,21 \\
\hline $4^{\circ}$ dia & $187,7 \pm 34,39$ & $170,3 \pm 22,69$ & 0,6 & 0,02 \\
\hline $5^{\circ} \mathrm{dia}$ & $185,1 \pm 32,87$ & $171,4 \pm 19,28$ & 0,5 & 0,04 \\
\hline
\end{tabular}

GKT (Grupo Kinesio Taping); GP (Grupo placebo). Nota: Teste t Student, ${ }^{*} \mathrm{p} \leq 0,05$.
Diferente do observado no Single-Leg Hop Test, o salto em distância revelou melhor resultado no grupo GKT em 4 dias de avaliação dos saltos, ou seja, do $2^{\circ}$ ao $5^{\circ}$ dia foram observados resultados significativos, conforme mostra a Tabela 4 .

Tabela 4. Comparação das médias de desempenho $(\mathrm{em} \mathrm{cm})$ no salto de impulso horizontal entre o grupo kinesio taping (GKT) e o grupo placebo (GP)

\begin{tabular}{|c|c|c|c|c|}
\hline \multirow{2}{*}{ Dias } & \multicolumn{2}{|c|}{ Salto em distância } & \multirow{2}{*}{$\begin{array}{c}\text { d de } \\
\text { Cohen }\end{array}$} & \multirow{2}{*}{ *p-valor } \\
\hline & GKT & GP & & \\
\hline $1^{\circ} \mathrm{dia}$ & $223,1 \pm 60,53$ & $210,6 \pm 28,65$ & 0,2 & 0,32 \\
\hline $2^{\circ} \mathrm{dia}$ & $235,6 \pm 58,64$ & $204,4 \pm 25,57$ & 0,6 & 0,005 \\
\hline $3^{\circ} \mathrm{dia}$ & $233,4 \pm 54,49$ & $204,6 \pm 25,16$ & 0,6 & 0,005 \\
\hline $4^{\circ}$ dia & $233,9 \pm 53,79$ & $210,3 \pm 25,69$ & 0,5 & 0,02 \\
\hline $5^{\circ} \mathrm{dia}$ & $228,7 \pm 49,21$ & $206,5 \pm 20,20$ & 0,5 & 0,01 \\
\hline
\end{tabular}

GKT (Grupo Kinesio Taping); GP (Grupo placebo).

Nota: Teste t Student; Teste Man-Whitney, ${ }^{*} \mathrm{p} \leq 0,05$.

Por fim, quando analisado o teste de impulsão vertical, constatou-se um padrão semelhante de desempenho observado no salto em distância. Houve melhor desempenho nos saltos do $2^{\circ}$ ao $5^{\circ}$ dia no grupo GKT quando comparado ao GP, com resultados estatisticamente significantes, conforme mostra a Tabela 5.

Vale ressaltar que no $5^{\circ}$ dia de cada salto, os resultados no GKT foram superiores ao GP, de forma significativa. Ou seja, neste momento os atletas já estavam há 24 horas sem usar a bandagem e, mesmo assim, ainda pode-se observar efeitos estimulantes que pudessem melhorar o desempenho dos saltos.

Tabela 5. Comparação das médias de desempenho $(\mathrm{em} \mathrm{cm})$ no salto vertical entre o grupo kinesio taping (GKT) e o grupo placebo (GP)

\begin{tabular}{|c|c|c|c|c|}
\hline \multirow{2}{*}{ Dias } & \multicolumn{2}{|c|}{ Teste de impulsão vertical } & \multirow{2}{*}{$\begin{array}{c}\text { d de } \\
\text { Cohen }\end{array}$} & \multirow{2}{*}{ *p-valor } \\
\hline & GKT & GC & & \\
\hline $1^{\circ} \mathrm{dia}$ & $101,7 \pm 10,61$ & $96,5 \pm 6,84$ & 0,5 & 0,08 \\
\hline $2^{\circ}$ dia & $102,5 \pm 9,89$ & $97,0 \pm 6,97$ & 0,6 & 0,05 \\
\hline $3^{\circ} \mathrm{dia}$ & $103,5 \pm 9,47$ & $97,5 \pm 7,00$ & 0,7 & 0,02 \\
\hline $4^{\circ} \mathrm{dia}$ & $103,7 \pm 20,0$ & $98,1 \pm 6,97$ & 0,3 & 0,04 \\
\hline $5^{\circ}$ dia & $101,7 \pm 21,00$ & $98,2 \pm 6,49$ & 0,2 & 0,22 \\
\hline
\end{tabular}

Nota: Teste t Student; Teste Man-Whitney, ${ }^{*} \mathrm{p} \leq 0,05$. 


\section{DISCUSSÃO}

Neste estudo a utilização da bandagem elástica sobre os músculos gastrocnêmios foi utilizada para identificar possíveis alterações na força muscular e melhora do desempenho físico, utilizando como parâmetros os saltos horizontais por meio do Single-Leg Hop Test e do salto em distância, e o salto vertical por meio do teste de impulsão vertical. O que pode ser justificado por Kase ${ }^{15}$, criador do método Kinesio Taping ${ }^{\circledR}$, a aplicação da bandagem elástica sobre a pele com finalidade de facilitação muscular otimiza a comunicação neural com os mecanorreceptores, aumentando o número de unidades motoras recrutadas durante a contração. Desta forma, quando sobreposta na musculatura alvo, há uma melhora na produção da força muscular corrigindo a função, ao reforçar músculos fracos ou inativos ${ }^{15,22,23}$.

As respostas desencadeadas pela bandagem elástica devem-se aos estímulos gerados principalmente pelas terminações de Ruffini que se adaptam a tensões diferentes sinalizando causas de deformação elástica na pele e dos tecidos adjacentes mais profundos ${ }^{10,24}$. Já os Corpúsculos de Paccini são estimulados por movimentos rápidos dos tecidos, vibrações e mudanças de pressão. Respondem em intervalos de tempo de centésimos de segundo e são importantes para detectar a vibração dos tecidos ou outras alterações rápidas do estado mecânico dos tecidos ${ }^{25,26}$.

A técnica utilizada neste estudo para o GKT visou a facilitação muscular por meio da aplicação da bandagem partindo da origem para a inserção muscular, sendo a bandagem elástica em formato de Y e com tensão predeterminada pela técnica Paper Off que significa utilizar a tensão normal da bandagem em torno de $15 \%$ a $25 \%$ no ventre muscular e estimular a epiderme aplicando a fita imediatamente após soltá-la do papel, pode ser pode ser observado o emprego desta técnica em outros estu$\operatorname{dos}^{17,27}$.

Para avaliar a efetividade da ação da bandagem nos membros inferiores, se fizeram necessários ajustes nos saltos propostos, a fim de evitar com que os membros superiores fossem capazes de influenciar positivamente nos saltos. Portanto, os atletas posicionaram os membros atrás do tronco de forma que ficassem contidos, favore- cendo a exigência somente os membros inferiores durante a realização dos testes. Gomes et al. ${ }^{28}$ observaram, em atletas de basquete e futebol, que o balanço dos braços interfere nos saltos, melhorando o desempenho do atleta e permitindo ganho de impulso; uma vez não utilizando o balanceio dos braços, é exigido do atleta maior velocidade de reação e contração nos membros inferiores para obtenção de força durante a fase concêntrica do ato muscular, assim como estes dados podem ser observados em outros estudos ${ }^{29}$.

Tendo tomado as precauções para tentar diminuir os possíveis vieses de avaliação, os resultados deste estudo mostraram que não houve relevância significativa na associação da aplicação da bandagem elástica e o aumento do rendimento físico dos atletas tanto do GKT quanto do GC ao longo dos cinco dias de avaliação, ou seja, os valores dos saltos não variaram ao longo dos dias após a aplicação da bandagem, o que pode ser justificado pela perda da aderência da bandagem em alguns atletas devido ao clima da região amazônica, caracterizado como quente e úmido, o que favorece a sudorese excessiva, prejudicando a permanência da bandagem por dias seguidos $^{30}$.

Mesmo apresentando dificuldade na permanência da bandagem em alguns atletas, quando se comparou os grupos no salto vertical e horizontais, o GKT após $24 \mathrm{~h}$ da aplicação da bandagem elástica demonstrou inserir efeitos proprioceptivos nos indivíduos com a técnica de facilitação, aumentando assim seus resultados $(\mathrm{em} \mathrm{cm})$ quando comparado ao GC. Fato este que demonstra a importância da aplicação da técnica original, pois a tensão sobre a pele imediatamente abaixo da bandagem pode otimizar a comunicação neural com os mecanorreceptores, aumentando o número de unidades motoras recrutadas durante a contração. Essas ações favorecem reflexos gerados pelo estiramento e encurtamento de tendões e músculos promovendo o aproveitamento da energia elástica armazenada conforme é citado em alguns estudos ${ }^{27,31}$.

O Single-Leg Hop Test demonstrou valores significativos para o grupo GKT somente no último dia de aplicação da bandagem e após a retirada desta, os resultados encontrados por este salto demonstram seguir outros estudos na literatura. Porém, Silva ${ }^{31}$, que utilizou o Kinesio Taping ${ }^{\circledR}$ na região do quadríceps em 24 sujeitos 
do sexo masculino, praticantes de atividades físicas irregularmente, dividindo-os em dois grupos, um que recebeu a aplicação da bandagem com tensão e outro sem a bandagem tensionada, aponta que os efeitos da KT durante dois dias, após $24 \mathrm{~h}$ e $48 \mathrm{~h}$, para realização de saltos horizontais, e dentre eles estava o Hop Test, mostrou que não houve diferença estatística entre os dois grupos, confrontando os resultados da presente pesquisa.

Este efeito dúbio pode ser explicado devido o Single-Leg Hop Test ser comumente utilizado em avaliações de lesões no joelho, para verificação de agilidade e propriocepção, além de aferição de força dos músculos quadríceps ${ }^{32-34}$. Neste estudo pode-se entender que a validade do teste não tenha sido realmente avaliada pois a bandagem não fora aplicada no quadríceps. E os gastrocnêmios não são mencionados na literatura como ator principal de impulsão para realização do salto, mas sim de como músculo de equilíbrio ${ }^{35}$. No entanto, o que pode ser explicado é que melhorando o equilíbrio muscular dos gastrocnêmios ao longo dos dias com a utilização do KT, a ação muscular do quadríceps possa ter sido potencializada, aumentando o desempenho muscular e melhorando o salto.

Diferente do Single-Leg Hop Test, o salto em distância utiliza de recursos dinâmicos como velocidade, impulsão e potência, assemelhando-se ao gesto do salto vertical e suas propriedades de alongamento e contração muscular para melhora do rendimento conforme citado em outros estudos ${ }^{29,35}$.

Sobre a importância dos saltos verticais em outros estudos, Huang et al. ${ }^{25}$, por sua vez, utilizaram a eletromiografia em indivíduos divididos em dois grupos, com a KT e com fita placebo. No estudo, os indivíduos realizaram saltos verticais com o propósito de se avaliar os músculos sóleo, gastrocnêmio e tibial anterior. Após 30 minutos de aplicação da KT, os sujeitos apresentaram maiores valores de forças e reação ao solo durante o salto vertical e maior ativação elétrica do gastrocnêmio, o que pode ser observado neste estudo com resultados satisfatórios no grupo GKT para o salto vertical ao longo dos dias de avaliação, perdendo sua efetividade quando retirada a bandagem.

Neste estudo reparou-se que nos saltos horizontais a partir de 3 metros e Single-Leg Hop Test, após a retirada da KT de ambos os grupos, o GKT demonstrou continuar variando significativamente em relação ao GC, enquanto no salto vertical não houve essa variação no último dia. Estudos sobre eletromiografia (EMG) demonstram que há manutenção dos efeitos da KT até $48 \mathrm{~h}$ após sua retirada ${ }^{36}$. Deste modo, observa-se nos rendimentos obtidos pelo GKT que a utilização da bandagem elástica pôde favorecer aqueles que receberam a técnica em relação ao grupo controle.

Embora se tenha observado resultados satisfatórios quanto à utilização da bandagem com o intuito de facilitação muscular com melhora do desempenho físico dos saltos, ainda há de se mencionar as limitações deste estudo que estão ligadas à manutenção da bandagem durante todo o processo de avaliação dos atletas. Sabe-se que Belém (PA) possui um clima quente e úmido, propício para sudorese excessiva, e assim facilitava a não adesão das fitas em alguns dos atletas. Mesmo realizando a troca imediata da bandagem, poderia ter levado a viés de coleta dos dados mascarando os reais efeitos do KT quando analisados e comparados seus efeitos ao longo dos dias. Assim, novas estratégias precisam ser tomadas para que se possa evitar perdas da fixação da bandagem durante todo o processo de avaliação dos saltos.

\section{CONCLUSÃO}

No presente estudo não houve aumento da potência dos membros inferiores no grupo GKT ao longo dos 5 dias analisados (24, 48 e 72 horas de aplicação do KT e 24 horas após a retirada). Porém, quando se comparou entre os grupos, o GKT apresentou resultados superiores na potência dos membros inferiores, no qual para o Single-Leg Hop Test houve aumento apenas 72 horas após a aplicação do KT e 24 horas após a retirada do mesmo. Para o salto em distância, houve aumento durante todos os dias de uso do KT e após a retirada da bandagem. Por fim, no teste de impulsão vertical houve apenas durante o uso do KT.

Estes dados podem ser estendidos à prática clí- 
nica permitindo elucidar que aqueles indivíduos que utilizam o KT podem aumentar a potência dos membros inferiores facilitando a contração muscular e melhorando a biomecânica corporal. E esta potência não está relacionada ao tempo prolongado do uso do KT e sim à técnica específica utilizada. Mesmo com este efeito positivo da bandagem é recomendável um bom condicionamento e preparo físico para melhora do gesto esportivo e acredita-se que estudos mais aprofundados com uso de outras tecnologias para verificar o real efeito muscular que a bandagem pode causar nestes músculos são importantes para embasar o que fora encontrado na presente pesquisa.

\section{REFERÊNCIAS}

1. Lopes PBL, Pereira Junior PP. A importância da tecnologia e da análise de jogo no voleibol. Revista de trabalhos acadêmicos. 2015(10):1-4.

2. Carvalho AC. Estudo Comparativo do Salto Vertical entre Desportistas especializados em Saltos e Não-Desportistas, de ambos os gêneros [monografia]. Porto: Faculdade de Desporto da Universidade do Porto; 2008. 70p.

3. Moura NA, Moura TFP, Borin JP. Buscando relações entre velocidade de abordagem e desempenho em saltos horizontais: estudo a partir de atletas de elite do troféu Brasil de atletismo 2003. Revista Brasileira de Ciência e Movimento. 2005; 13(2): 7-15.

4. Ferreira IC. A Importância do trabalho de impulsão vertical em voleibolistas como diferencial dos atletas de alto nível [trabalho de conclusão de curso]. Formiga: Centro Universitário de Formiga - UNIFOR; 2013. 18p.

5. Mcginnis PM. Biomecânica do Exporte e do Exercício. Porto Alegre: Artmed; 2015.

6. Felicissimo CT, Dantas JL, Moura ML, Moraes AC. Respostas neuromusculares dos membros inferiores durante o protocolo intermitente de saltos verticais em voleibolistas. Motriz: Revista de Educação Física. 2012; 18(1): 153-64.

7. Marchetti PH, Freitas FS. Efeitos do Kinesio Taping no desempenho de força e na atividade muscular: uma breve revisão. Rev CPAQV. 2016; 8(1): 1-21.

8. Lumbroso D, Ziv E, Vered E, Kalichman L. The effect of kinesio tape application on hamstring and gastrocnemious muscles in healthy young adults. J Bodywork and Movement Therapies. 2013; 18(1): 130-8.

9. Lemos TV, Albino AC, Matheus JP, Barbosa Ade M. The effect of kinesio taping in foward bending of the lumbar spine. J Phys Ther Sci. 2014; 26(9): 1371-75.

10. Araújo GJS, Rodrigo AS, Cavalcante MLC, Moraes MRB. A aplicabilidade do recurso Kinesio Taping ${ }^{\circledR}$ nas lesões desportivas: uma revisão de literatura. Revista Pesquisa em Fisioterapia. 2014; 4(3): 189-96.

11. Zhang S, Fu W, Pan J, Wang L, Xia R, Liu Y. Acute effects of kinesio taping on muscle strength and fatigue in the forearm of tennis players. Journal of Science and Medicine in Sport. 2016; 19(6): 459-64.

12. Parreira PCS, Costa LCM, Hespanhol Junior LC, Lopes AD, Costa LOP. Current evidence does not support the use of Kinesio Taping in clinical practice: a systematic review. Journal of Physiotherapy. 2014; 60: 31-9.

13. Ackland TR, Elliott BC, Bloomfield J. Anatomia e Biomecânica aplicadas no esporte. São Paulo: Manole; 2011.

14. Lemos TV, Pereira KC, Protássio CC, Lucas LB, Matheus JPC. The effect of Kinesio Taping on handgrip strength. J Phys Ther Sci. 2015; 27(3): 567-70.

15. Kase K, Lemos TV, Dias EM. Kinesio Taping ${ }^{\circledR}$ introdução ao método e aplicações musculares. São Paulo: Andreoli; 2013.

16. Branco BHM, Andreato LV, Ribeiro ED, Oliveira HG, Almeida FN, Nardo Junior N. Development of tables of classifying judô atheletes according to maximal isometric strength and muscular power, and comparisons between athletes at diferente competitive levels. Sport Sciences for Health. (Testo Stampato). 2018: 1-8.

17. Martins RC. Análise das variáveis dinâmicas dos saltos verticais [monografia]. Belo Horizonte: Escola de Educação Física, Fisioterapia e Terapia Ocupacional - Universidade Federal de Minas Gerais; 2009. 26p.

18. Silva T, Ribeiro F, Venâncio J. Comparação da per- 
formance funcional do membro inferior entre jovens futebolistas e jovens não treinados. Fisioter. Mov. 2010; 23(1): 105-12.

19. 19 Sawle L, Freeman J, Marsden J. Intra-rater reliability of the multiple single leg hop-stabilization test and relathionships with age, leg dominance and training. Int J Sports Phys Ther. 2017; 12(2): 190-98.

20. Coledam DHC, Arruda GA, Oliveira AR. Efeitos de um programa de exercícios no desempenho de crianças nos testes de flexibilidade e impulsão vertical. Motriz: Revista de Educação Física. 2012; 18(3): 515-25.

21. Rhea MR. Determining the magnitude of treatment effects in strength training research through the use of the effect size. Journal of Strength and Conditioning Research. 2004; 18(4): 918-20.

22. Artioli DP, Bertolini GRF. Kinesio Taping: aplicação e seus resultados sobre a dor: revisão sistemática. Fisioterapia e Pesquisa. 2014; 21(1): 94-9.

23. González-Iglesias J, Fernández-de-Las-Peñas C, Cleland JÁ, Del Rosario Gutiérrez-Veja M. Short-term effects of cervical kinesio taping on pain and cervical range of motion in patients with acute whiplash injury: a randomized clinical Trial. J Orthop Sports Phys Ther. 2009; 39(7): 515-21.

24. Williams S, Whatman C, Hume PA, Sheerin K. Kinesio Taping in treatment and prevention of sports injuries: a meta-analysis of the evidence for its effectiveness. Sports Med. 2012; 42(2): 152-64.

25. Huang CY, Hsieh TH, Lu SC, Su FC. Effect of Kinesio tape to muscle activity and vertical jump performance in healthy inactive people. Biomed Eng Online. 2011; 10:70.

26. Nakajima MA, Baldridge C. The effect of Kinesio ${ }^{\circledR}$ Tape on vertical jump and dynamic postural control. Int J Sports Phys Ther. 2013; 8(4): 393-406.

27. Menzel HJ, Campos CE. Análise do squat jump e counter movement jump através de testes motores e biomecânicos. In: Silami-Garcia E, Lemos KL, Greco PJ (org). Temas Atuais em Educação Física e Esportes IV. Belo Horizonte: Health, 1999, 57-68.

28. Gomes MM, Pereira G, Freitas PB, Barela JA. Características cinemáticas e cinéticas do salto vertical: com- paração entre jogadores de futebol e basquetebol. Rev Bras Cineantropom Desempenho Hum. 2009; 11(4): 392-9.

29. Zanchet MA, Del Vecchio FB. Efeito da Kinesio taping sobre força máxima e resistência de força em pedalistas. Fisioter Mov. 2013; 26(01): 115-21.

30. Csapo R, Alegre LM. Effects of Kinesio $®$ Taping on skeletal muscle strength - A meta - analysis of current evidence. J Sci Med Sport. 2015; 18(4): 450-6.

31. Silva JRF. Influências da Kinesio Taping no desempenho do Salto em Distância, em Indivíduos sadios jovens [monografia]. Brasília: Universidade de Brasília; 2014. 37p.

32. Micha DN, Ferreira M. Física no Esporte - parte 1: saltos em esportes coletivos. Uma motivação para o estudo da mecânica através da análise dos movimentos do corpo humano a partir do conceito de centro de massa. Revista Brasileira de Ensino de Física. 2013; 35(3): 1-9.

33. Firth BL, Dingley P, Davies ER, Lewis JS, Alexander $\mathrm{CM}$. The effect of kinesiotape on function, pain, and motoneuronal excitability in healthy people and people with Achilles tendinopathy. Clin J Sport Med. 2010; 20(6): 416-21.

34. Slupik A, Dwornik M, Bialoszewski D, Zych E. Effect of Kinesio Taping on bioelectrical activity of vastus medialis muscle. Preliminary report. Ortop Traumatol Rehabil. 2007; 9(6): 644-51.

35. Duarte F, Alca DV, Gesser ES, Krebs FG, Rempel C. Avaliação da potência muscular de membros inferiores após realização de protocolo de treinamento neuromuscular e de força muscular. ConScientia e Saúde. 2009; 8(3): 405-13.

36. Magalhães IEJ. Influência do uso da Kinesiotaping nas respostas funcionais e eletrofisiológicas em homens fisicamente ativos [dissertação]. Brasília: Universidade de Brasília; 2015. 134p.

Recebido em: 15/08/2018

Aceito em: 09/10/2018 